\title{
INTERTEMPORAL CHOICE AND THE CROSS-SECTIONAL VARIANCE OF MARGINAL UTILITY
}

\author{
Orazio P. Attanasio and Tullio Jappelli*
}

\begin{abstract}
The theory of intertemporal choice predicts that the crosssectional variance of the marginal utility of consumption is equal to its own lag plus a constant and a random component. Using general preference specifications and some assumptions about the nature of the random component, we provide an explicit test of this hypothesis. Our approach circumvents the necessity to identify a pure age profile of the crosssectional variance of consumption and yields a well-specified statistical test. This test is applied to data from the United States, the United Kingdom, and Italy. The results are remarkably consistent with the restrictions implied by the theory of intertemporal consumption choices.
\end{abstract}

\section{Introduction}

$\mathrm{O}$ ne remarkable prediction of the permanent-income hypothesis with certainty equivalence is that consumption inequality within a group of households with fixed membership should, on average and over long periods of time, increase with age. By this model, the change in individual consumption represents the annuity value of the revisions in labor income, which (under rational expectations) are unpredictable. Then, if income shocks are not perfectly correlated within the group, the cross-sectional variance of consumption increases with age until retirement (that is, until uncertainty is resolved). This prediction of the theory was first investigated by Deaton and Paxson (1994), who estimate the age profile of the cross-sectional variance of consumption using average cohort data for the United States, the United Kingdom, and Taiwan and find that consumption inequality increases with age in all three countries.

If certainty equivalence is relaxed, the permanent-income hypothesis provides predictions about the marginal utility of consumption, and not consumption itself. In particular, the model does not generate an explicit relation between age and consumption inequality or, for that matter, between consumption and income. We thus propose to focus directly on the Euler equation for consumption, and consider the time-series properties of the cross-sectional variance of an approximation of the marginal utility of consumption rather than consumption. Under the identifying assumptions discussed in section II, the theory implies that, in a regression of the cross-sectional variance of the marginal utility of

Received for publication May 28, 1998. Revision accepted for publication May 23, 2000.

* University College London, Institute for Fiscal Studies, CEPR, and NBER; and CSEF, University of Salerno, and CEPR, respectively.

We would like to thank James Banks, Richard Blundell, Luigi Pistaferri, and Guglielmo Weber for useful comments and suggestions, and Sarah Tanner for help with the U.K. data. Particular thanks are owed to Angus Deaton and Christina Paxson for interesting discussions and extensive exchanges that we hope improved the paper. We retain responsibility for any errors. This paper is part of the research project, "Structural Analysis of Household Savings and Wealth Positions over the Life-Cycle." The Training and Mobility of Researchers Network Program (TMR) of the European Commission DGXII and the Italian National Research Council (CNR) provided financial support. consumption on a constant and its own lag, the coefficient of the latter is unity. We test this hypothesis in this paper. Our procedure does not require certainty equivalence; does not impose assumptions regarding the relation between age, time, and cohort effects in estimating the age profile of the cross-sectional variance; and delivers a simple statistical test of a well-specified null hypothesis that allows us to apply standard inference tools.

The benefits are not cost free, however. The test we propose is based on assumptions regarding the properties of the residuals of the Euler equation. A rejection of the null hypothesis could therefore be due to the failure of these assumptions and not necessarily of the permanent income model. Furthermore, because we relax certainty equivalence, we cannot exploit the relation between consumption, income innovations, and age. For instance, we cannot check if the increase in consumption inequality slows around retirement, as implied by the certainty equivalence case. Nor can we attribute the spreading of consumption inequality to permanent and transitory changes in uncertainty, as Blundell and Preston (1998) propose.

We use average cohort data to test our hypothesis. In the absence of long panel data on consumption, these data are particularly well suited to the problem at hand. Although we cannot compute the cross-sectional variance of the same group of individuals over time, average cohort data allow us to track the variance of a representative sample of the same cohort. Another advantage of cohort data is that they allow us to apply an appropriate instrumental variables estimator.

The empirical analysis, presented in section III, uses three approximations to the marginal utility of consumption. Initially, we measure marginal utility as the log of expenditure on nondurable goods. We then take into account the lifecycle variations of family size and define marginal utility as the log of nondurable consumption expenditure per adult equivalent. Finally, we compute marginal utility by relying on available estimates of the parameters of a flexible utility function. These parameters have been estimated with the same data sets used in this paper. Having estimated the marginal utility for each household in the sample, we can then compute the cross-sectional variance of marginal utility of population groups defined by year of birth and test whether the coefficient of lagged variance on current variance is unity. The advantage of the third procedure is that marginal utility is allowed to depend upon a full set of demographic and labor supply variables.

We use three sets of cohort data. The British Family Expenditure Survey (1974-1993) and the U.S. Consumer Expenditure Survey (1980-1995) cover a sufficiently long timespan and allow consistent estimation of an Euler equa- 
tion for consumption. We also use data drawn from the Italian Survey of Household Income and Wealth (19871995); because this is too short a timespan to provide reliable estimates of the Euler equation, in this case we do not attempt to measure marginal utility by using a flexible utility function. Section IV summarizes the results.

\section{The Cross-Sectional Variance of Marginal Utility}

We take off from Deaton and Paxson (1994) and their exploration of the implications of the permanent-income hypothesis for the evolution of consumption and income inequality. It is well known that, if the utility function is quadratic and if the real interest rate equals the discount rate, optimal consumption for individual $i$ follows a martingale:

$$
c_{i, t+1}=c_{i, t}+\epsilon_{i, t+1}
$$

The cross-sectional variance of the variables in equation (1) is

$$
\operatorname{var}\left(c_{i, t+1}\right)=\operatorname{var}\left(c_{i, t}\right)+\operatorname{var}\left(\epsilon_{i, t+1}\right)+\operatorname{cov}\left(c_{i, t}, \epsilon_{i, t+1}\right),
$$

in which variances and covariances are computed over a cross section of households whose composition is constant over time. If aggregate variables are part of the information set of each agent, Deaton and Paxson (1994) show that the time average of $\operatorname{cov}\left(c_{i, t}, \epsilon_{i, t+1}\right)$ is zero. Equation (2) then implies that, in a group with fixed membership, the variance of consumption of individuals aged $t$ is stochastically dominated by the variance of consumption of the same individuals aged $t+1$. In other terms, in a stationary population, consumption inequality increases with age on average and over long periods of time. Because $\epsilon_{i, t+1}$ represents the annuity value of the innovations in permanent income, consumption inequality increases until individuals face income shocks. Such dispersion should at least slow down for older households if earnings shocks dominate interest-rate shocks.

\section{A. Relaxing Certainty Equivalence}

Equation (1) and (2) can be generalized to more-flexible preference specifications. Suppose that utility is intertemporally separable and that the instantaneous utility function, defined over nondurable consumption, depends on a set of conditioning variables $z$ (demographic and labor supply, let us say), and on an unobservable component, $v$, that captures unobserved heterogeneity. In this case, it can be immediately shown that the Euler equation that applies to individual $i$ is

$$
\begin{aligned}
E_{t} & {\left[\mu\left(c_{i, t+1}, \theta^{\prime} z_{i, t+1}, v_{i, t+1}\right)\left(1+r_{t+1}^{k}\right) \beta\right] } \\
& =\mu\left(c_{i, t}, \theta^{\prime} z_{i, t}, v_{i, t}\right)
\end{aligned}
$$

where $\mu(\cdot)$ is the marginal utility of consumption, $r_{t+1}^{k}$ is the rate of return on a generic asset $k$, $\theta$ is a set of parameters of the utility function, and $\beta=1 /(1+\delta)$ is the discount factor.

Note that both $\theta$ and $\beta$ are assumed to be time invariant and common to all individuals.

Inspection of equation (3) reveals that, if one abandons the assumption of quadratic utility in favor of more-general preference specifications, it becomes very difficult to derive a relationship between the cross-sectional variance of the level of consumption and age. Before investigating in detail the implications of the general specification in equation (3) and discussing our approach, it is useful to consider some simple examples in which the lifecycle model does not necessarily imply that the cross-sectional variance of consumption increases with age. Deaton and Paxson (1994) also discuss some of these situations.

A simple situation in which, even with quadratic utility, the cross-sectional variance of utility does not necessarily increases over time is when the discount factor differs from the rate of interest. The Euler equation is then $c_{i, t+1}=$ constant $+(1+\delta) /(1+r) c_{i, t}+\epsilon_{i, t}$. If $r>\delta$, then consumption increases on average over the lifecycle, reinforcing the effect of the variance of the innovation term. If instead $r<\delta$, consumption declines over the lifecycle. In the absence of income shocks, the cross-sectional variance of consumption would then also fall with age. Income innovations may partly or completely offset the tendency of the variance to fall with age. Note that, with uncertain lifetimes, the risk of longevity makes consumers effectively more impatient, raising the possibility of a declining consumption path, particularly in old age.

Another simple example in which the variance of consumption does not necessarily increase is one with no uncertainty and isoelastic utility, so that the Euler equation is $\ln c_{i, t+1}=\ln c_{i, t}+\sigma(r-\delta)$, where $\sigma$ is the intertemporal rate of substitution. Clearly, although $\operatorname{var}\left(\ln c_{i, t+1}\right)$ is constant for each age group, $\operatorname{var}\left(c_{i, t+1}\right)$ increases with age if $r>\delta$ and declines if $r<\delta$.

The situation in which there is no uncertainty is equivalent, as far as the cross-sectional variance of consumption is concerned, to a situation in which individual shocks are perfectly insured. As with perfect insurance, the only shocks to consumption are aggregate ones, and the cross-sectional variance of the marginal utility of consumption is constant over time. Whether the level of cross-sectional variance of consumption increases depends on the nature of preferences and on the relative size of interest rate and discount factor. For instance, with isoelastic preferences and the interest rate less than the discount factor, the cross-sectional variance of consumption levels declines with age. ${ }^{1}$

\footnotetext{
${ }^{1}$ These first two examples indicate that, if one were interested in checking whether the cross-sectional variance of consumption increases over time, it might be more useful to focus on the variance of log consumption rather than on the variance of consumption levels. The
} 
As discussed by Deaton and Paxson (1994), another case in which the lifecycle pattern of the cross-sectional variance of consumption is not obvious is one in which demographic (or other variables) affect the marginal utility of consumption. If, for instance, utility is defined in terms of per capita, rather than total consumption, with quadratic preferences, the Euler equation takes the form $c_{i, t+1} / N_{i, t+1}=c_{i, t} / N_{i, t}+$ $\epsilon_{i, t}$. The lifecycle pattern of the cross-sectional variance of consumption depends now not only on the cross-sectional variance of the shocks but also on the cross-sectional variability (and its changes over time) in family size. ${ }^{2} \mathrm{~A}$ similar argument can be made with respect to any agerelated preference shift (the $z$ variables in equation (3)).

The final example is one with isoelastic utility and noninsurable shocks. If the distribution of consumption growth is log normal, one obtains an exact Euler equation with a term for precautionary saving:

$$
\begin{aligned}
\ln c_{i, t+1}= & \ln c_{i, t}+\sigma(r-\delta)+\left(\sigma^{-1} / 2\right) \\
& \times\left[\operatorname{var}\left(\Delta \ln c_{i, t+1}\right)\right]+\epsilon_{t+1},
\end{aligned}
$$

where $\sigma^{-1}$ is the coefficient of relative prudence and $\epsilon_{t+1}$ an expectational error. ${ }^{3}$ Computing the cross-sectional variance of both sides of the Euler equation, one sees that the spreading of consumption depends also on the age evolution of the conditional variance of consumption growth. We know very little about this term and how it varies across consumers as they age, which thus makes the predictions of the model hard to pin down.

The examples show that, with slight departures from the simplest model with certainty equivalence, consumption inequality can increase or decrease with age, depending on demographics, preferences, and the gap between the discount and interest rates. It should also be clear that the age pattern of consumption levels is not the same as that of consumption logs. The same applies to the marginal utility of consumption. Looking again at equation (3) one sees that the cross-sectional variance of marginal utility declines with age for precisely the same reasons for which one expects the variance of consumption to increase with age.

Even though the relationships between age and the variances of consumption levels, log consumption, and marginal utility are ambiguous, the theory does have predictions about the dynamics of consumption inequality. Our approach consists, therefore, in focusing on the time-series

former is a better approximation of marginal utility when preferences are isoelastic. Furthermore, because consideration of the log of consumption implicitly implies the consideration of a $\log$ linearization, the result is independent of the relative size of interest rate and discount factor. Deaton and Paxson focus mainly on the pattern of the variance of log consumption.

${ }^{2}$ The cross-sectional variance of the number of adult equivalents (used in the later empirical exercises) is flat in the very first part of the lifecycle, increases in middle age, and decreases in the last part of the lifecycle. The same is true for the variance of the logs of adult equivalents.

${ }^{3}$ The variance in this Euler equation is the time-series variance of consumption growth for each consumer. autocorrelation of the cross-sectional variance of the marginal utility of consumption. As an example, consider again equation (2). One can see that a regression of the crosssectional variance of consumption-proportional to the marginal utility of consumption under quadratic utility-on its lagged value should yield, under some conditions, a coefficient of unity.

To see how this point extends to more-general situations such as equation (3), we consider the case wherein the instantaneous utility function is isoelastic, that is:

$$
U(c, z, v)=\frac{c_{i, t}^{1-\sigma^{-1}}}{1-\sigma^{-1}} \exp \left[-\sigma^{-1}\left(\theta^{\prime} z_{i, t}-v_{i, t}\right)\right] .
$$

The Euler equation (3) can now be expressed as a linear function of the logarithm of consumption:

$$
\begin{aligned}
& \ln \left(c_{i, t+1}\right)+\theta^{\prime} z_{i, t+1}-v_{i, t+1} \\
&= \sigma \ln \beta+\ln \left(c_{i, t}\right)+\theta^{\prime} z_{i, t}-v_{i, t}+\tilde{\phi}_{i, t+1} \\
&+\sigma \ln \left(1+r_{t+1}^{k}\right)+\tilde{\epsilon}_{i, t+1} \\
&= \sigma \ln \beta+\ln \left(c_{i, t}\right)+\theta^{\prime} z_{i, t}-v_{i, t}+\phi_{i} \\
&+\sigma \ln \left(1+r_{t+1}^{k}\right)+\epsilon_{i, t+1},
\end{aligned}
$$

where $\sigma$ is the elasticity of intertemporal substitution,

$\tilde{\boldsymbol{\epsilon}}_{i, t+1}$ is an expectational error, and

$\tilde{\phi}_{i, t+1}$ is the higher conditional moments of the distribution of consumption in equation (3). ${ }^{4}$

The second equality in equation (4) follows by defining $\epsilon_{i, t+1}=\tilde{\phi}_{i, t+1}-\phi_{i}+\tilde{\epsilon}_{i, t+1}$, where $\phi_{i}$ indicates the time-series average of the conditional moments. The crosssectional variance of the two sides of equation (4) is

$$
\begin{aligned}
\operatorname{var} & \left\lfloor\ln \left(c_{i, t+1}\right)+\theta^{\prime} z_{i, t+1}+v_{i, t+1}\right\rfloor \\
= & \operatorname{var}\left[\ln \left(c_{i, t}\right)+\theta^{\prime} z_{i, t}+v_{i, t}\right]+\operatorname{var}\left(\phi_{i}\right) \\
& +\operatorname{var}\left(\epsilon_{i, t+1}\right)+2 \operatorname{cov}\left[\ln \left(c_{i, t}\right), \phi_{i}+\epsilon_{i, t+1}\right] .
\end{aligned}
$$

Equation (5), as such, cannot be measured empirically. (For one thing, the parameters $\theta$ are unknown. Second, the variable $v$ is by definition unobservable. Finally, $\phi$ includes unknown parameters and moments of a distribution that has not yet been specified.) But equation (5) does highlight a sharp prediction of the theory: that the coefficient of the lagged cross-sectional variance of marginal utility equals 1.5 It is precisely because of this property that, in the simple

\footnotetext{
${ }^{4}$ For instance, if the interest rate is constant and consumption growth is normally distributed, this term equals the conditional variance of consumption growth.

${ }^{5}$ The left side of equation (4) is not exactly the marginal utility of consumption, as we have divided by $\sigma$, the elasticity of intertemporal substitution. If constant across individuals, however, this term represents only a factor of proportionality that does not affect the arguments below. For simplicity, we continue to refer to the left side of equation (4) as the marginal utility.
} 
case of quadratic utility, the cross-sectional variance of consumption increases, on average, with age. In the remaining portion of this section, we illustrate how we propose to test this important implication of intertemporal optimization.

To make equation (5) operational, we need to make a number of identifying assumptions. We deal with the first problem (that the parameter vector $\theta$ is unknown) below. For the present, we assume that $\theta$ is known. To address the fact that $v$ and $\phi$ are unobservable, one can write equation (4) as

$$
\begin{aligned}
& \ln \left(c_{i, t+1}\right)+\theta^{\prime} z_{i, t+1} \\
& =\sigma \ln \beta+\ln \left(c_{i, t}\right)+\theta^{\prime} z_{i, t}+\phi_{i} \\
& \quad+\sigma \ln \left(1+r_{t+1}^{k}\right)+\Delta v_{i, t+1}+\epsilon_{i, t+1} .
\end{aligned}
$$

Assuming that the real interest rate is common across households, the cross-sectional variance of the two sides of equation (6) is given by the following expression:

$$
\begin{aligned}
\operatorname{var} & \left\lfloor\ln \left(c_{i, t+1}\right)+\theta^{\prime} z_{i, t+1}\right\rfloor \\
= & \operatorname{var}\left\lfloor\ln \left(c_{i, t}\right)+\theta^{\prime} z_{i, t}\right\rfloor \\
& +\operatorname{var}\left(\phi_{i}+\Delta v_{i, t+1}+\epsilon_{i, t+1}\right) \\
& +2 \operatorname{cov}\left[\ln \left(c_{i, t}\right)+\theta^{\prime} z_{i, t}, \phi_{i}-\Delta v_{i, t+1}+\epsilon_{i, t+1}\right] .
\end{aligned}
$$

Only the first term on the right side can be measured empirically using cross-sectional data. However, the second and third terms can be decomposed into a part that is constant over time (such as the variance of $\phi$ and its covariance with the other components) and a time-variant component. Equation (7) can then be rewritten as

$$
\begin{aligned}
\operatorname{var}[ & \left.\ln \left(c_{i, t+1}\right)+\theta^{\prime} z_{i, t+1}\right] \\
= & \omega_{0}+E\left(\omega_{t+1}\right)+\operatorname{var}\left[\ln \left(c_{i, t}\right)+\theta^{\prime} z_{i, t}\right] \\
& +\omega_{t+1}-E\left(\omega_{t+1}\right) \\
= & \varpi_{0}+\operatorname{var}\left[\ln \left(c_{i, t}\right)+\theta^{\prime} z_{i, t}\right]+\varpi_{t+1},
\end{aligned}
$$

where $E\left(\omega_{t+1}\right)$ denotes the time series average of $\omega_{t+1}$;

$\omega_{0}$ includes the time-invariant terms of the right side of equation (7):

$$
\begin{aligned}
\omega_{0}= & \operatorname{var}\left(\phi_{i}\right)+2 \operatorname{cov}\left[\ln \left(c_{i, t}\right)+\theta^{\prime} z_{i, t}, \phi_{i}\right] \\
& +2 \operatorname{cov}\left[\phi_{i}, \Delta v_{i, t+1}\right]+2 \operatorname{cov}\left(\phi_{i}, \epsilon_{i, t+1}\right) ; \text { and }
\end{aligned}
$$

$\omega_{t+1}$ denotes the time-varying components of the crosssectional variance:

$$
\begin{aligned}
\omega_{t+1}= & \operatorname{var}\left(\Delta v_{i, t+1}\right)+\operatorname{var}\left(\epsilon_{i, t+1}\right)+2 \operatorname{cov}\left[\ln \left(c_{i, t}\right)\right. \\
& \left.+\theta^{\prime} z_{i, t}, \Delta v_{i, t+1}\right]+2 \operatorname{cov}\left(\Delta v_{i, t+1}, \epsilon_{i, t+1}\right) .
\end{aligned}
$$

The terms $\varpi_{0}$ and $\varpi_{t+1}$ are implicitly defined by the second equality in equation (8). Note that most components of $\varpi_{t+1}$ do not vanish. An exception is $\operatorname{cov}\left(\phi_{i}, \epsilon_{i, t+1}\right)$, which is 0 under the permanent income hypothesis.

\section{B. The Empirical Test}

Suppose now that we can identify one or more population groups whose membership is fixed over time. The crosssectional variances in equation (8) can then be estimated by computing the corresponding sample variances within groups at different time periods. This application of synthetic panel techniques yields consistent estimates of the corresponding population variances. We can then run the regression

$$
\begin{aligned}
\operatorname{var} & {\left[\ln \left(c_{i, t+1}\right)+\theta^{\prime} z_{i, t+1}\right] } \\
& =\varpi_{0}+\pi \operatorname{var}\left[\ln \left(c_{i, t}\right)+\theta^{\prime} z_{i, t}\right]+\varpi_{t+1}
\end{aligned}
$$

and test the hypothesis that $\pi=1$.

The use of OLS to estimate equation (9) would produce inconsistent estimates of $\pi$, however, for at least three reasons. First, the hypothesis that the cross-sectional covariance of $\Delta v_{i, t+1}$ with the observed component of marginal utility at time $t$ is 0 is likely to be violated. Second, the time varying-terms included in $\varpi_{t+1}$ might be correlated over time with the variance term in equation (8). Finally, in a finite sample, one never observes the true population moments, but only an estimate of these moments that are therefore affected by measurement error. This induces a further source of bias in the estimate of $\pi$.

Thus, the issue is that of finding an instrument for $\operatorname{var}\left[\ln \left(c_{i, t}\right)+\theta^{\prime} z_{i, t}\right]$ that is uncorrelated with $\varpi_{t+1}$ and with the sampling error. Synthetic cohort data suggest an ideal candidate for dealing with the potential bias arising from measurement error: if the samples used in estimating the variances in equation (9) are independent-as they typically are in repeated cross sections-so are the sample errors of subsequent periods. Therefore, $\operatorname{var}\left[\ln \left(c_{i, t-1}\right)+\right.$ $\left.\theta^{\prime} z_{i, t-1}\right]$ is, as far as sampling error is concerned, a valid instrument for the corresponding variance at time $t$ with which it is obviously correlated. However, to guarantee that the (twice) lagged variance is a valid instrument overall, one must assume that $\operatorname{cov}\left\{\varpi_{t+1}\right.$, var $\left.\left[\ln \left(c_{i, t-1}\right)+\theta^{\prime} z_{i, t-1}\right]\right\}=$ 0 , that is, that the twice-lagged variance is uncorrelated with the deviation of $\omega_{t+1}$ from its unconditional mean. This is a strong assumption that deserves further scrutiny.

To better understand the issues involved, it is worthwhile to strip the model down to its simplest version, the one with certainty equivalence behind equation (1) and (2), wherein $\omega_{t+1}=\operatorname{var}\left(\epsilon_{i, t+1}\right)+\operatorname{cov}\left(c_{i, t}, \epsilon_{i, t+1}\right)$. If aggregate shocks are part of the agents' information set, the second term is a genuine innovation and therefore its covariance (over time) with the lagged cross-sectional variance is 0 under the permanent-income hypothesis. The assumption that $\operatorname{var}\left(c_{i, t}\right)$ is uncorrelated with the deviations of $\operatorname{var}\left(\epsilon_{i, t+1}\right)$ 
from its time mean, however, is not an implication of the permanent-income hypothesis. Rather, its validity depends on the nature of the shocks $\epsilon_{i, t+1}$, on the agents' information set as well as on the market structure in which they operate.

Suppose that the expectational error of the Euler equation is described by $\epsilon_{i, t+1}=\alpha_{i} \psi_{t+1}+\eta_{i, t+1}$ where $\psi_{t+1}$ is an aggregate shock and $\eta_{i, t+1}$ is an idiosyncratic shock assumed to be i.i.d. across individuals and over time and independent of $\alpha_{i}$ and $\psi_{t+1}$. (Notice that the aggregate shock is allowed to affect consumers in different fashion.) This representation of the error term encompasses several models. ${ }^{6}$

Given the assumed structure of the error term, $\operatorname{var}\left(\epsilon_{i, t+1}\right)=\psi_{t+1}^{2} \operatorname{var}\left(\alpha_{i}\right)+\operatorname{var}\left(\eta_{i, t+1}\right)$. If $\eta_{i, t+1}$ is indeed i.i.d., then $\operatorname{var}\left(\eta_{i, t+1}\right)$ is constant over time. If $\psi_{t}$ is also i.i.d.-so that $E\left(\psi_{t+1}^{2}\right)$ is constant over time$\operatorname{cov}\left[\operatorname{var}\left(\epsilon_{i, t+1}\right)\right.$, var $\left.\left(c_{i, t}\right)\right]=0$, and $\operatorname{var}\left(c_{i, t-1}\right)$ is obviously a valid instrument for $\operatorname{var}\left(c_{i, t}\right)$. If instead $\psi_{t}$ is heteroskedastic and/or $\psi_{t+1}^{2}$ exhibits a considerable amount of persistence, then $\operatorname{var}\left(c_{i, t-1}\right)$ may not be a valid instrument for $\operatorname{var}\left(c_{i, t}\right)$. For instance, if the conditional time variance of $\psi_{t+1}$ follows an $\mathrm{ARCH}(1)$ process, $\operatorname{cov}\left[\operatorname{var}\left(\epsilon_{i, t+1}\right), \operatorname{var}\left(c_{i, t}\right)\right]=0$, and our instrument is still a valid one; but, if $\psi_{t+1}^{2}$ evolves according to an ARCH of order 2 or higher (or as any GARCH process), it is not. Similar arguments apply to $\eta_{i, t+1}$ and to its cross-sectional variance.

Although we develop the previous example with quadratic utility, the same considerations apply to the more general model that relaxes certainty equivalence. In particular, when the innovations to the Euler equation can be decomposed into an aggregate shock and an individualspecific shock, the period $t-1$ variance of the marginal utility of consumption is a valid instrument for its period $t$ variance if

- the aggregate shock is part of the agents' information set,

- its second moments do not exhibit persistence over time, and

- the evolution of the cross-sectional variance of the idiosyncratic shocks is not correlated with the crosssectional variance of consumption.

These are not predictions of the permanent-income/lifecycle model. Thus, our test that $\pi=1$ is a joint test of the model and these additional assumptions. A finding that $\pi \neq 1$ cannot therefore be taken necessarily as a contradiction of the model, but a finding that $\pi=1$ implies that the model and our additional assumptions are consistent with the data.

We also experiment with an alternative instrument for $\operatorname{var}\left[\ln \left(c_{i, t}\right)+\theta^{\prime} z_{i, t}\right]$. Theory suggests that, in the case of

\footnotetext{
${ }^{6}$ In the case of complete contingent markets, for instance, the error term has the factor structure $\epsilon_{i, t+1}=\psi_{t+1}+\eta_{i, t+1}$, where $\eta_{i, t+1}$ is i.i.d and independent of $\psi_{t+1}$.
}

certainty equivalence, the cross-sectional variance of consumption is an increasing function of age, so that age is likely to be correlated with the dependent variable even relaxing certainty equivalence. However, for age to be a valid instrument, one must assume that it is uncorrelated with the cross-sectional variance of $\eta_{i, t}$ and with the time variance of $\psi_{t}$. The latter assumption is quite plausible. The former, however, is questionable, especially when one considers periods of the lifecycle during which uncertainty changes in a dramatic and yet systematic way. The most obvious example is retirement. For this reason, in the empirical section we use age both in addition to and in place of the lagged variance as an instrument for the current variance. We also check if our estimates of $\pi$ are sensitive to the inclusion of the retired in our samples.

Notice that the hypothesis that $\pi=1$ holds both under the lifecycle model with uninsurable shocks and under perfect insurance. In this sense, our approach (unlike Deaton and Paxson's (1994)) cannot be used to discriminate between these two models. However, not all models imply such a relationship. To see why this is the case, consider a model in which some consumers might be liquidity constrained. For a generic consumer $i$ at time $t$, the Euler equation for consumption is

$$
\begin{aligned}
& \mu\left(c_{i, t}, z_{i, t}, v_{i, t}\right) \\
& \quad=E_{t}\left[\mu\left(c_{i, t}, z_{i, t}, v_{i, t}\right)(1+r) /(1+\delta)\right]+\lambda_{i, t}
\end{aligned}
$$

where $\lambda_{i, t}$ is the Kuhn-Tucker multiplier associated with the liquidity constraint for consumer $i$ at time $t$. Therefore, this term equals 0 for those consumers that are not liquidity constrained. If one thinks that such multipliers are related, when positive, to the level of income, one sees that the cross-sectional variance of consumption is related not only to its lagged value, but also to the value of the crosssectional variance of income. ${ }^{7}$ Furthermore, as the number of liquidity-constrained consumers is likely to vary over the business cycle, one would not expect the coefficient of the lagged variance of consumption to be unity.

\section{Measuring Marginal Utility and Its Cross-Sectional Variance}

The problem that the parameter vector $\theta$ is unknown can be handled in several ways. The simplest strategy is to drop the $z$ variables and regress the variance of log consumption on the lagged variance. A more satisfactory measure of marginal utility is to assume that $z$ includes only family size and to scale the consumption data using adult equivalent weights. This is equivalent to assuming that the utility

\footnotetext{
${ }^{7}$ Another model that does not predict $\pi=1$ is the myopic model of consumption, $c_{i, t}=y_{i, t}$. One sees immediately that $\pi=0$ here and that the cross-sectional variance of consumption is entirely explained by the cross-sectional variance of income.
} 
function is defined on consumption per adult equivalent, rather than on consumption,

$$
U(c, P, N)=\frac{N_{i, t}}{1-\sigma^{-1}}\left(\frac{c_{i, t}}{P_{i, t}}\right)^{1-\sigma^{-1}}
$$

and estimate

$$
\begin{aligned}
\operatorname{var} & {\left[\ln \left(\frac{c_{i, t+1}}{P_{i, t+1}}\right)+\sigma \ln \left(\frac{P_{i, t+1}}{N_{i, t+1}}\right)\right] } \\
& =\varpi_{0}+\pi \operatorname{var}\left[\ln \left(\frac{c_{i, t}}{P_{i, t}}\right)+\sigma \ln \left(\frac{P_{i, t}}{N_{i, t}}\right)\right]+\varpi_{i+1}
\end{aligned}
$$

where $P_{i, t}$ is the number of adult equivalents, $N_{i, t}$ is the number of household's members, and $\sigma$ is the elasticity of intertemporal substitution. ${ }^{8}$

A third possibility is to posit more-flexible preference specifications that explicitly allow the utility function to depend on labor supply and demographic variables. Because the preference parameters are unknown, they have to be estimated from the data. We propose the following approach. In a first stage, we use the same cohort data and exploit the orthogonality conditions implied by the Euler equation to estimate the parameters of the utility function. In a second stage, we use the estimated parameters to compute the cross-sectional variance in equation (9) and to test the hypothesis that $\pi=1$. In section III, rather than performing the first stage, we rely on previous estimates. In particular, we use preference parameters estimated by Euler equations that have been fitted to the same data sets used in this paper. ${ }^{9}$ In all cases, we estimate equation (9) and (10) for all cohorts simultaneously. Because the population groups (cohorts) might be characterized by different variances in $\phi$ and $v$, we always check if the results are sensitive to the introduction of dummies for cohort-specific intercepts.

As discussed in subsection IIB, in the presence of (occasionally) binding liquidity constraints, the cross-sectional variance of $(\log )$ consumption would not only depend on its lagged value but also on the cross-sectional variance of the Kuhn-Tucker multiplier associated with the constraints. As such multipliers are likely to be related to current income, we test for the presence of the variance of lagged income into our regression equations.

\footnotetext{
${ }^{8}$ The second term in the square brackets in equation (10) appears if utility is defined in terms of consumption per adult equivalent but is then summed over the number of family members rather than adult equivalents. With log utility, the expression simplifies to one in which consumption per capita enters the marginal utility. In practice, the inclusion of the second term does not make much difference for the results we obtain.

${ }^{9}$ A more efficient strategy, which we do not pursue here, might be to consider equation (6) and (9) as a simultaneous equations system. This would be equivalent to estimating the parameters of the utility function exploiting the restrictions that the theory implies on the time series of both first and second cross-sectional moments. One could then use the overidentifying restrictions (including $\pi=1$ ) to test the model. This procedure would also have the advantage of explicitly taking into account the fact that the vector $\theta$ is unknown.
}

\section{Comparison with Deaton and Paxson's Methodology}

Average cohort data lend themselves very well to the problem at hand. By definition, birth cohorts are groups with fixed membership. ${ }^{10}$ The most intuitive way of testing the implications of equation (5) is to track the crosssectional variance of the marginal utility as the cohort ages. Indeed, in their seminal contribution, Deaton and Paxson (1994) constructed average cohort data for the United States, the United Kingdom, and Taiwan, considering the graphical representation of the cross-sectional variance and testing the hypothesis that the variance of $(\log )$ consumption increases with age.

Their methodology therefore involves identifying an age profile of the cross-sectional variance. This amounts to disentangling age, time, and cohort effects. Because these variables are perfectly collinear (time $=$ cohort + age), Deaton and Paxson normalize the time effects to 0 , so that the evolution over time of the cross-sectional variances of each cohort is explained only by a combination of age and cohort effects. ${ }^{11}$ Without structural and/or out-of-sample information, this normalization is indispensable: Any time trend can be written as a combination of age and cohort effects, and any age effect can be written as a combination of year and cohort effects.

The normalization is essentially an issue of interpretation when describing average cohort profiles of consumption or wages. In the case at hand, however, the implication of the theory being tested is of a structural relationship between age and the cross-sectional variance, and the assumption that all variance trends are explained by cohort and age effects is not particularly appealing. This problem is particularly relevant for the sample period that Deaton and Paxson consider, at least for the United States and the United Kingdom. During the 1980s, there was a considerable increase in inequality that affected all cohorts and was partly related to the increase in the return to education. Our approach completely circumvents this identification issue by focusing on the implication that the coefficient on the lagged variance (that is, the coefficient $\pi$ in equation (9)) is equal to unity. A related advantage is that it tests a welldefined null hypothesis against which standard inference tools can be applied.

\footnotetext{
${ }^{10}$ To be more precise, Deaton and Paxson (1994) assume that time effects have zero mean and are orthogonal to a linear trend. This allows identification of all coefficients of interest. They also assume that age, time, and cohort effect do not interact. We are abstracting here from differential mortality and immigration. Because our samples are not restricted to couples, we also abstract from differential divorce rates.

${ }^{11}$ A slightly less restrictive assumption would be that the average of the time dummies in the sample period is 0 and that the time dummies are orthogonal to a time trend. This is in fact equivalent to assuming that all the trends in the data can be interpreted as a combination of age and cohort effects and therefore, by definition, predictable. As Angus Deaton has pointed out to us, this normalization is similar to the normalization that is used when accounting for seasonality, when the coefficient of one of the seasonal dummies is set to 0 . In our opinion, the only difference is that, in the seasonality example, one normalizes the level of the variable under study, while in the present context one normalizes its level and changes.
} 
Another important difference between our approach and Deaton and Paxson's is that focusing on the autocorrelation properties of the cross-sectional variance of the marginal utility avoids the problems that are caused by the fact that, in many plausible situations (some of which we discussed in subsection IIA), the cross-sectional variance of consumption does not increase with time. Our approach lends itself naturally to the consideration of preference specifications that are more flexible than quadratic, such as those that allow for a precautionary saving motive. Furthermore, we do not need to assume any relationship between the interest rate and the discount factor and/or the constancy over time of the former.

Our approach, however, is not without disadvantages. First of all, we can obtain consistent estimates of the coefficient in equation (9) only under a number of assumptions that were discussed at length in subsections IIA and IIB. Furthermore, unlike Deaton and Paxson's approach, ours does not identify the lifecycle profile of consumption inequality and therefore cannot be used to discriminate between a model with perfect insurance and a standard lifecycle model with uninsurable individual shocks. Because of this, we can say that our approach is a complement to-rather than a substitute for-Deaton and Paxson's.

\section{Empirical Results}

We present our empirical evidence in three parts. We first describe the data sets used, then provide a graphical illustration of the main feature of the cross-sectional variance of consumption and marginal utility, and finally report the results of our econometric analysis. Some of the details of the data treatment are given in appendix A.

\section{A. Data Description}

We estimate equation (9) and (10) using three sets of average cohort data. The U.K. Family Expenditure Survey is the largest dataset, including twenty annual surveys (1974-1993). The U.S. Current Expenditure Survey is available for sixteen years (1980-1995). The Italian Survey of Household Income and Wealth is available from 1984 to 1995, but, given the characteristics of this survey, we use data from only 1987 to 1995 . Furthermore, the Italian data is available every other year, so that we can use a total of five surveys. Our choice to focus on the United States, the United Kingdom, and Italy was guided mainly by the availability of cohort data for these countries.

The FES: The main motivation for the collection of the Family Expenditure Survey (FES) on the part of the U.K. Department of Employment is the computation of the weights for the retail price index. In recent years, the data set has been used extensively to describe the behavior of U.K. households and to estimate structural models of consumption behavior. The sample includes approximately
7,000 households per year. (The survey has no panel element.) Each household stays in the sample for two weeks, during which it compiles a diary with its expenditures. At the end the diary is collected, and further information on various expenditure items during the previous three months (typically durable goods and utilities) is gathered. The survey also takes information on several economic and demographic household characteristics, ranging from labor supply to household composition. The quality of the consumption data (thanks especially to the use of the diary method rather than retrospective interviews) is remarkably good. ${ }^{12}$

As each household stays in the sample for just two weeks, consumption figures are heavily affected by seasonality. This problem is particularly serious for the cross-sectional variances we compute. For instance, part of the difference in consumption between a respondent interviewed in December and one interviewed in August certainly is due to seasonal effects and not to genuine cross-sectional variability. To account for this, we deseasonalize the individual consumption observations before computing the cross-sectional variance. The seasonal adjustment is described in appendix A.

The CEX: The Consumer Expenditure Survey (CEX) is run by the U.S. Bureau of Labor Statistics for the same reasons as the FES (namely to compute the consumer price index), and the size of the sample is roughly similar (approximately 7,000 households per year). Like the FES, the CEX also collects data on income, demographics, and several other variables. ${ }^{13}$ There are, however, two important differences between the two. First, the CEX has a short panel element, in that each household is interviewed five times over fifteen months. The first interview is only a contact interview on which no information is disclosed in the public-use tape; in each of the subsequent four interviews, however, the household reports detailed information on the expenditures incurred during each of the three previous months. Potentially, that is, each household provides twelve monthly expenditure accounts. Second, the households in the CEX do not compile a diary but answer retrospective questions.

The households in the CEX do not complete their cycle of interviews simultaneously. Because interviews are performed every month, households interviewed in different months report information on different periods. For exam-

\footnotetext{
${ }^{12}$ A recent study edited by Banks and Johnson (1997) compares expenditure and income data in the FES with those of the national accounts. It finds that-controlling for differences in definitions (especially of housing services), reference population, and sampling structure-the FES data track very well their counterparts in the national accounts.

${ }^{13}$ Some questions are asked only in the last or in the first and last interview. In addition, the timing of some variables is different from that of expenditure. Most notably, the consumption information refers to each of the three months preceding the interview, although the income and labor supply questions (asked only in the second and fifth interviews) refer to the twelve months preceding the interview.
} 
ple, a household that completes its last interview in January 1990 provides information on each of the twelve months of 1989, and a household that completes its cycle of interviews in June 1990 covers the period from June 1989 to May 1990. One-twelfth of the sample is replaced each month, and of course not all households complete the cycle of five interviews.

This sampling structure complicates the computations of the cross-sectional variances in which we are interested. We cannot compute the cross-sectional variance in a given year using the monthly observations as we do in the FES because several observations would refer to a single household. Variation across monthly observations would therefore reflect time-series (seasonal or cyclical) rather than crosssectional variability. Estimating annual consumption for each household is complicated by the overlapping structure of the sample: the "year" over which we have information for each household would be different depending on the month in which the household completes its cycle of interviews. Annual estimates are further complicated by the fact that not all households complete the cycle.

We consider two possible methods of constructing the cross-sectional variances. The first is to estimate the "calendar year" consumption figure for each household on the basis of the months available, with seasonal adjustment. By this method, most households would provide figures to be used in the computation of the variance in two different years, and this should be taken into account in the choice of instruments. The second procedure involves considering only one monthly observation per household; after seasonal adjustment one can compute the cross-sectional variance for all the households in a given quarter or year.

We use the second procedure. Its drawback, however, is that, by limiting itself to one observation per household, it wastes useful information. On the other hand, it is simple to apply and yields a sample whose structure is very similar to that of the FES. To minimize the effect of nonrandom attrition, the monthly observation we select is the first available. The procedure for seasonal adjustment is the same one used for the FES (detailed in appendix A).

The SHIW: The primary purpose of the Bank of Italy Survey of Household Income and Wealth (SHIW) is to collect detailed data on demographics and households' income and wealth. It uses a representative sample of the Italian resident population, and probability selection is enforced at every stage of sampling. Like the FES and the $\mathrm{CEX}$, it samples the household, defined as all persons residing in the same dwelling and related by blood, marriage, or adoption. The SHIW consumption data are collected by retrospective questions on broad categories of durable and nondurable consumption during the previous year. This implies that seasonal adjustment is not necessary. Given the different purpose of the survey, the detail and quality of the consumption data is not as high as that in the
FES or the CEX. Nonetheless, the SHIW consumption data match the trends in the national accounts data reasonably well. The sample size is slightly larger than that of the other two surveys (approximately 8,000 households).

Until 1984, the age of the household head is available only in wide bands precluding the construction of year-ofbirth cohorts. In 1986, the data for expenditures on durable goods is indistinguishable from nondurable consumption. Since 1987, the survey was conducted every other year. For our purpose, therefore, the only usable surveys are the five surveys taken from 1987 to 1995.

\section{B. Measures of Marginal Utility}

In all three surveys, we define consumption as total household expenditure on nondurable goods. This excludes expenditures on durable goods, health, education, and housing-except in the case of the SHIW, where it excludes durable expenditure only. Our focus on nondurable consumption implies, implicitly, an assumption of separability with respect to the excluded components. Current values are transformed into constant values using a consumer price index. As discussed in section II, we use three different approximations of the marginal utility of consumption. The first is simply the logarithm of consumption, and the second is the log of consumption per adult equivalent. The third filters the consumption data using the preference parameters estimated by Attanasio and Weber (1993) for the United Kingdom and by Attanasio and Weber (1995) for the United States. The definition of adult equivalent used in the second procedure assigns a weight of 1 to the first adult, 0.8 to any additional adult, and 0.25 to any child. To compute the expressions in equation (10), it is also necessary to know the value of the elasticity of intertemporal substitution. Those reported in the following tables correspond to a value of $\sigma$ of 0.8. We experimented with alternative specifications, both for the definition of adult equivalent and for the assumption about the elasticity of intertemporal substitution, and we obtained very similar results. ${ }^{14}$

The SHIW allows a straightforward estimation of the variance of annual log consumption. The sample size and structure of the FES and CEX allow us to construct quarterly data. The results for the United Kingdom and the United States using annual observations are similar to those presented in the following subsections and can thus be omitted for brevity. The only notable difference is that the standard errors for the United States based on annual observations are slightly larger than those based on quarterly observations.

\section{Cohort Definition and Descriptive Analysis}

In each survey, we restrict the sample to households that are headed by individuals born between 1910 and 1959 and

\footnotetext{
${ }^{14}$ For brevity, these are not reported.
} 
Table 1.-Average and Minimum Cell Size in the FES, the CEX, and the SHIW

\begin{tabular}{|c|c|c|c|c|c|c|c|c|}
\hline \multirow[b]{2}{*}{ Cohort } & \multirow[b]{2}{*}{ Year of birth } & \multirow{2}{*}{$\begin{array}{c}\text { Age in } \\
1985\end{array}$} & \multicolumn{2}{|c|}{ United Kingdom (FES) } & \multicolumn{2}{|c|}{ United States (CEX) } & \multicolumn{2}{|c|}{ Italy (SHIW) } \\
\hline & & & Mean & Minimum & Mean & Minimum & Mean & Minimum \\
\hline 1 & 1910-1914 & $71-75$ & 136 & 68 & 58 & 22 & 384 & 363 \\
\hline 2 & 1915-1919 & $66-70$ & 127 & 81 & 71 & 35 & 390 & 298 \\
\hline 3 & 1920-1924 & $61-65$ & 161 & 125 & 84 & 49 & 714 & 661 \\
\hline 4 & 1925-1929 & $56-60$ & 142 & 102 & 83 & 52 & 799 & 766 \\
\hline 5 & 1930-1934 & $51-55$ & 134 & 106 & 81 & 46 & 836 & 807 \\
\hline 6 & 1935-1939 & $46-50$ & 141 & 114 & 87 & 54 & 918 & 856 \\
\hline 7 & 1940-1944 & $41-45$ & 151 & 118 & 107 & 61 & 859 & 838 \\
\hline 8 & 1945-1949 & $36-40$ & 183 & 134 & 143 & 91 & 938 & 874 \\
\hline 9 & $1950-1954$ & $31-35$ & 156 & 58 & 167 & 127 & 765 & 739 \\
\hline 10 & 1955-1959 & $26-30$ & 139 & 33 & 192 & 127 & 696 & 582 \\
\hline
\end{tabular}

The table reports the average and minimum cell size in the Family Expenditure Survey (FES), the Consumer Expenditure Survey (CEX), and the Survey of Household Income and Wealth (SHIW) by year of birth. The cells are used to compute the variance of log consumption.

define ten groups: cohort 1 includes those born in 19101914 , cohort 2 those born in 1915-1919, and so on, up to cohort 10 from 1955-1959. We define the age of the cohort as the median age of each cohort in a given sample period. (For instance, the age of cohort 1, born in 1910-1914, is 68 in 1980, 69 in 1981, and so on.)

Table 1 reports the cohort definition and, for each survey, the mean and minimum cell size by cohort. The assumption behind our procedure is that the cross-sectional variance refers to the same group of individuals at different points in time. Even though we do not need to observe the same individuals over time, the test requires that the sample composition be constant. The positive correlation between survival probabilities and wealth implies that rich households are overrepresented in the older cohorts. The correla- tion between wealth and young headship (young working adults living independently are likely to be wealthier than average) suggests that rich households may also be overrepresented in the youngest cohorts. Accordingly, we exclude households in which the head is older than eighty or younger than twenty.

The construction of cohort data involves taking means of the marginal utility within each of the cells over successive time periods. Aggregation, however, is not an issue in this context. Each of the methods of measuring the marginal utility of consumption first defines an index of marginal utility at the household level; the cross-sectional variance of this index can then be readily computed within each cell.

Before presenting the regression results, we provide a graphical exposition of the data. In figure 1 , we plot the

TABLE 2.-REgRessions FOR THE VARIANCE OF LOG CONSUMPTION

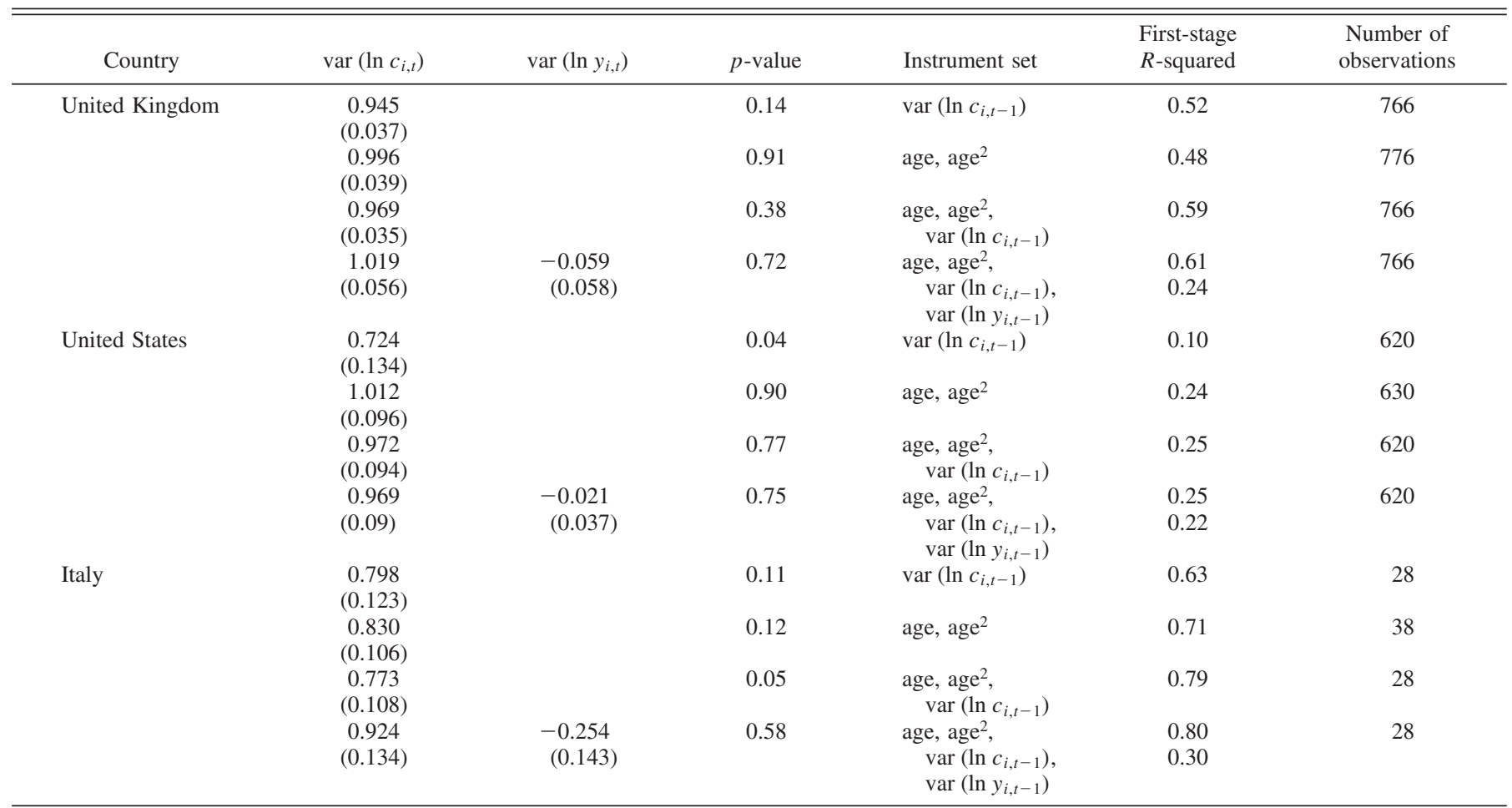

The table reports coefficient estimates of the regression var $\left\lfloor\ln \left(c_{i, t+1}\right)\right\rfloor=\varpi_{0}+\pi$ var $\left\lfloor\ln \left(c_{i, t}\right)\right\rfloor+\varpi_{t+1}$. The $p$-value refers to the test that $\pi$ is significantly different from 1 . The two $R$-squared in the fourth specification for each country refer to the first-stage regressions for the lagged variance of consumption and for the lagged variance of income, respectively. Standard errors are reported in parenthesis. 
Figure 1.-The Variance of Log Consumption

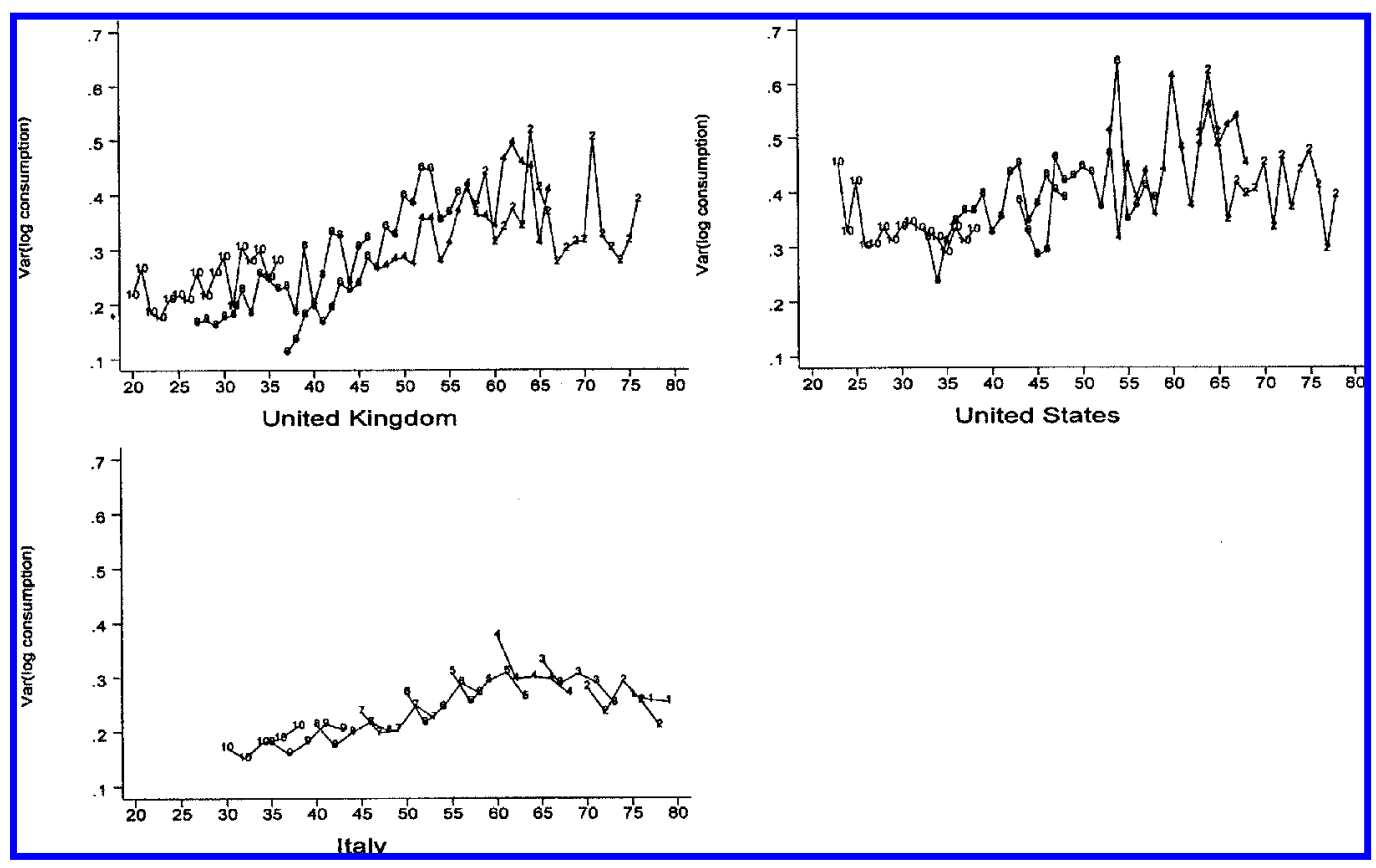

The figure plots the variance of the logarithm of nondurable consumption in the United Kingdom, the United States, and Italy. In the case of the United Kingdom and the United States, we plot the data for only cohorts 2 (born 1915-1919), 4 (1925-1929), 6 (1935-1939), 8 (1945-1949) and 10 (1955-1959).

cross-sectional variance of the logarithm of consumption for the United Kingdom, the United States, and Italy, respectively. Each connected segment refers to a different cohort, observed over time at different ages. The cohort numbers, let us recall, run from 1 (the oldest) to 10 (the youngest). To facilitate the comparison across countries, the graphs are plotted on the same scale. These graphs are similar to the raw variances produced by Deaton and Paxson (1994). (See their figures 2 and 3 for the United States and the United Kingdom, respectively.) $)^{15}$

As we stress in section II, interpreting these graphs is not easy because cohort, age, and time are perfectly collinear variables. Their distinct effects are not identified: for instance, any interpretation of the data in terms of age and cohort effects (normalizing time effects to 0 ) can be recast in terms of an alternative decomposition in terms of age and time effects (normalizing cohort effects to 0). Nonetheless, some features of the raw data are highly suggestive.

Interestingly, the absolute level of the variance is considerably higher in the United States ( 0.4 on average), lowest in Italy (0.25), and intermediate in the United Kingdom (0.3), reflecting very different patterns of consumption inequality in these countries. In the United Kingdom, there is some evidence of cohort effects, especially for households born after the second world war (cohorts 7 to 10 in figure 1). In the United States and Italy, cohort effects are absent or

${ }^{15}$ As mentioned, in the estimation for the United Kingdom and the United States, we use the variance of quarterly $(\log )$ consumption. To have legible pictures, we plot the variance only for the first quarter of each year and for five cohorts $(2,4,6,8$, and 10). weak at best. In the United Kingdom and Italy, the variance of log consumption increases up to age sixty and diminishes for older households, which Deaton and Paxson interpret as evidence in support of models with finite horizon, in which income shocks cease after retirement. The graph for the United States, by contrast, is much flatter over the entire lifecycle. ${ }^{16}$

In figure 2, we plot the cross-sectional variance of $\log$ consumption defined in terms of adult equivalent, assuming an intertemporal elasticity of substitution of 0.8. (See equation (10).) The behavior of these variances is rather different than in the previous figures in all three countries. First of all, the variance is now considerably smaller, especially in the United Kingdom. Second, the age pattern is much flatter than in the previous set of figures, suggesting that variation in household size and composition over the lifecycle explains a good part of the age profile of the cross-sectional variance of consumption. In the United Kingdom and the United States, there is no evidence of the spreading of consumption over the lifecycle. In Italy, the cross-sectional variance declines slightly with the age of the household head, reversing the pattern in figure $1 .{ }^{17}$

\footnotetext{
16 The pattern of inequality may reflect also differences in measurement of consumption in the different surveys.

${ }^{17}$ Using a flexible specification of preferences to impute marginal utility further reduces the cross-sectional variance in the United Kingdom and the United States. There is again weak evidence of age effects in consumption inequality only in the United Kingdom. For brevity, these figures are not reported.
} 
Figure 2.-The Variance of Log Consumption Per Adult Equivalent

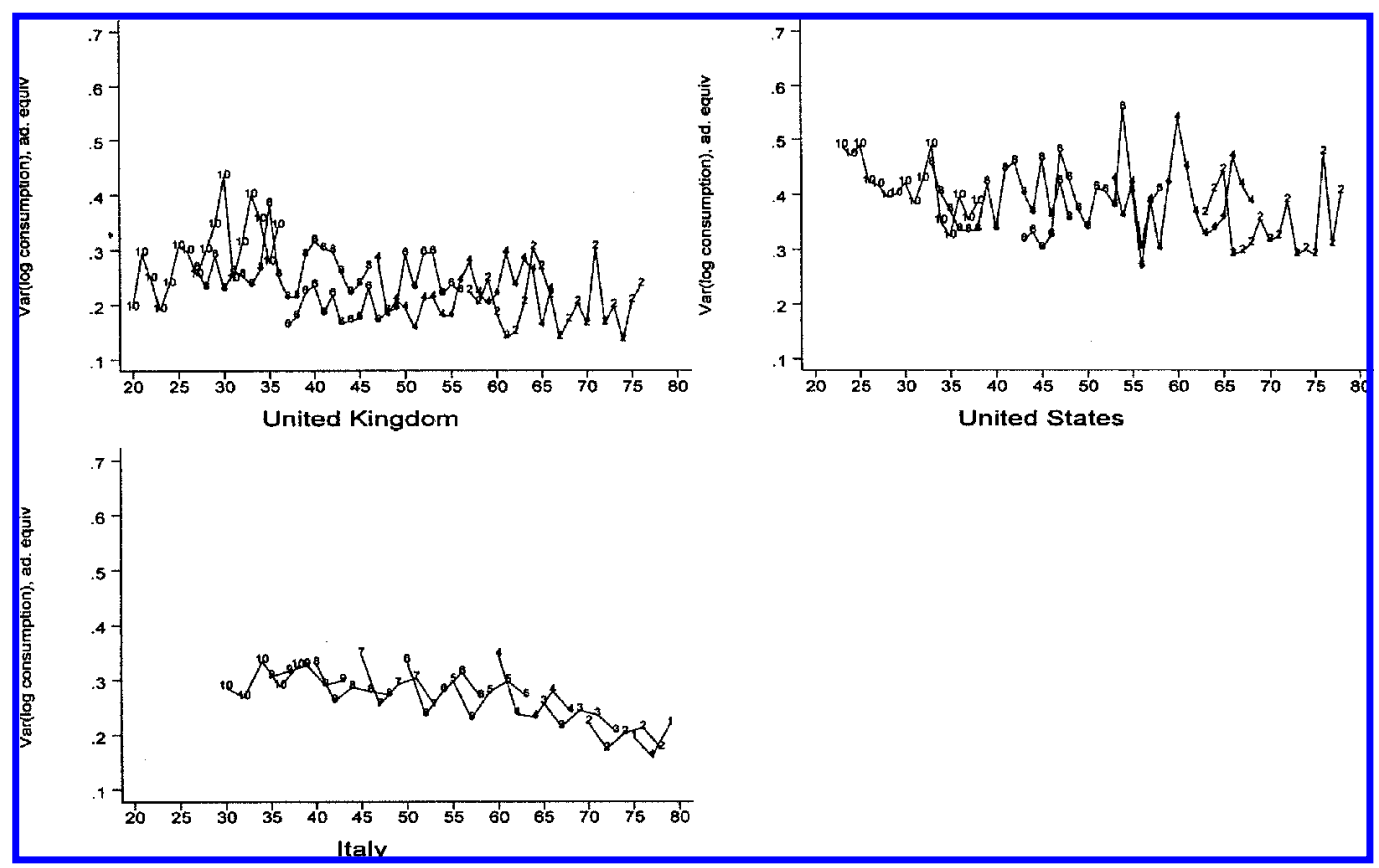

The figure plots the variance of the logarithm of consumption expenditures per adult equivalent in the United Kingdom, the United States, and Italy. Adult equivalent are computed assigning a weight of 1 to the first adult, 0.8 to any additional adult, and 0.25 to each child. In the case of the United Kingdom and the United States, we plot the data for only cohorts 2 (born 1915-1919), 4 (1925-1929), 6 (1935-1939), 8 (1945-1949) and 10 (1955-1959).

The last set of figures summarizes the age effect in the cross-sectional variance according to the different measures of marginal utility that are available in each of the three countries. The age effect is obtained by regressing the cross-sectional variance against a fifthorder age polynomial, a full set of cohort dummies, and a set of time dummies constrained to sum to 0 and to be orthogonal to a time trend. ${ }^{18}$ The estimated age profiles show that consumption inequality increases with age only for the variance of $\log$ consumption. Even in this case, however, the age effects show little spreading of consumption. Figure 3 shows that in the United Kingdom, the variance increases only from 0.2 to 0.4 , in the United States from 0.35 to 0.5 , and in Italy from 0.15 to 0.30 . If instead consumption is defined in terms of adult equivalent or if we directly impute marginal utility, we find flat (or even declining) age profiles of the cross-sectional variance in each of the three countries.

The pattern of the estimated age effects contrasts slightly with the findings of Deaton and Paxson, particularly in the case of the United States. Using the CEX, they find that the age effects in the United States increase from approximately 0.2 to 0.5 (while ours grows from 0.35 to 0.5 ) for both the variance of the logarithm of consumption and the variance computed defining consumption per adult equivalent. Part of the difference may derive from differences in the treat-

\footnotetext{
${ }^{18}$ This procedure differs slightly from that used by Deaton and Paxson because we use a polynomial in age rather than age dummies. This, however, does not affect our results in any substantive way.
}

ment of the data or different sample periods. ${ }^{19}$ They also use quarterly cells, but limit the analysis to the 1980-1990 period. However, computing the age effects after dropping the 1991-1995 years does not change the profiles in figure 3.

As we discussed in section II, only under special assumptions does the theory deliver a closed-form solution for consumption and allow a test of the hypothesis that consumption inequality increases with age on average and over long periods of time. Furthermore, such a prediction of the theory is testable only by arbitrarily normalizing the time effects. Using Deaton and Paxson's methodology, this prediction of the theory is rejected in each of the three data sets when we take into account the fact that part of the inequality in consumption over the lifecycle is explained by variation in household size and composition. We now turn to our alternative - and, in some respects, more general-test of the implication of intertemporal choices for the evolution of the cross-sectional variance of the marginal utility of consumption.

\section{Regression Results}

We regress the variance of log consumption on its own lag (see equation (9)) for the three countries for which we have data. Table 2 reports the point estimates, the standard

\footnotetext{
${ }^{19}$ Before computing the cross-sectional variance, we adjust our consumption figures using a model that allows monthly seasonal effects. Furthermore, to avoid complicated MA structures due to the overlapping structure of the sample as well as biases induced by nonrandom attrition, we use only the first interview completed by each household.
} 
Figure 3.-Estimated Age EfFects



The figure plots the estimated age effects of the variance of marginal utility in the United Kingdom, the United States, and Italy. The marginal utility is defined as log consumption, log consumption per adult equivalent, and filtering consumption with a flexible specification for preferences. Each profile is obtained by the fitted values of a regression of the variance of marginal utility on a fifth-order age polynomial, a full set of cohort dummies, and a set of time dummies restricted to sum to 0 and to be orthogonal to a time trend. The number of observations is 776 in the United Kingdom, 630 in the United States, and 38 in Italy.

errors, the $p$-value of a test that the coefficient of the lagged variance equals 1 , and the $R$-square of the first-stage regression. The basic specification for the variance of marginal utility also included a full set of cohort dummies. However, an $F$-test for the significance of the cohort dummies was never statistically different from 0 , regardless of the measure of marginal utility. The cohort dummies are therefore dropped in the final specifications.

As is shown in section II, OLS estimates of equation (9) are potentially subject to measurement error and endogeneity bias. Thus, for each specification, we report four sets of results. The first three regressions differ in instruments used. The first uses only the second lag of the cross-sectional variance; the second replaces the lagged variance with the (median) age and age square of the cohort; and the third includes age, age square, and the second lag of the variance. Finally, in the fourth line for each country and definition of marginal utility, we augment the regression with the lagged variance of household disposable income (instrumented with its second lag). Along with the estimate and standard error of the coefficient on the lagged variance of consumption (and income, when relevant), we report the $p$-value of a test of the hypothesis that $\pi=1$ and the $R$-square of the first-stage regression.

None of the regressions reported in table 2 rejects the null hypothesis that the coefficient of the lagged variance equals 1 , with the exception of the third regression for Italy, where the test rejects the null hypothesis at the five-percent level. The coefficients of the lagged variance are generally esti- mated with small standard errors, particularly in the case of the United Kingdom. Furthermore, the point estimates for the United States and the United Kingdom are remarkably close to 1 and similar across different sets of instruments. Note also that, in each regression, the instruments have explanatory power for var $\left(\ln c_{i t}\right)$.

Our results are not affected by excluding the youngest and oldest cohorts or cohorts whose cells are not very large. For instance, excluding retired households (older than 57 years) does not affect the estimate of $\pi$, regardless of the instruments that are used in the estimation.

As discussed in section II, the lagged cross-sectional variance of income might be relevant if earnings are related to the Kuhn-Tucker multipliers associated to liquidity constraints. The fourth row for each country in table 2 shows that there is no evidence that this term plays a role in the regression for the cross-sectional variance of consumption.

In table 3 , the dependent variable is the cross-sectional variance of $\log$ consumption per adult equivalent, as in equation (10). ${ }^{20}$ The estimates are not as precise as those in table 2, particularly for the United States, but the general pattern is confirmed. The magnitude of the estimated coefficients for the United Kingdom and the United States is hardly affected, although in Italy the point estimates are

\footnotetext{
${ }^{20}$ When the utility function is defined in terms of adult equivalent, marginal utility depends on the elasticity of intertemporal substitution. The results in the tables refer to the case in which the parameter equals 0.8 . Different values yield similar results.
} 
Table 3.-Regressions for the Variance of Log Consumption Per Adult Equivalent

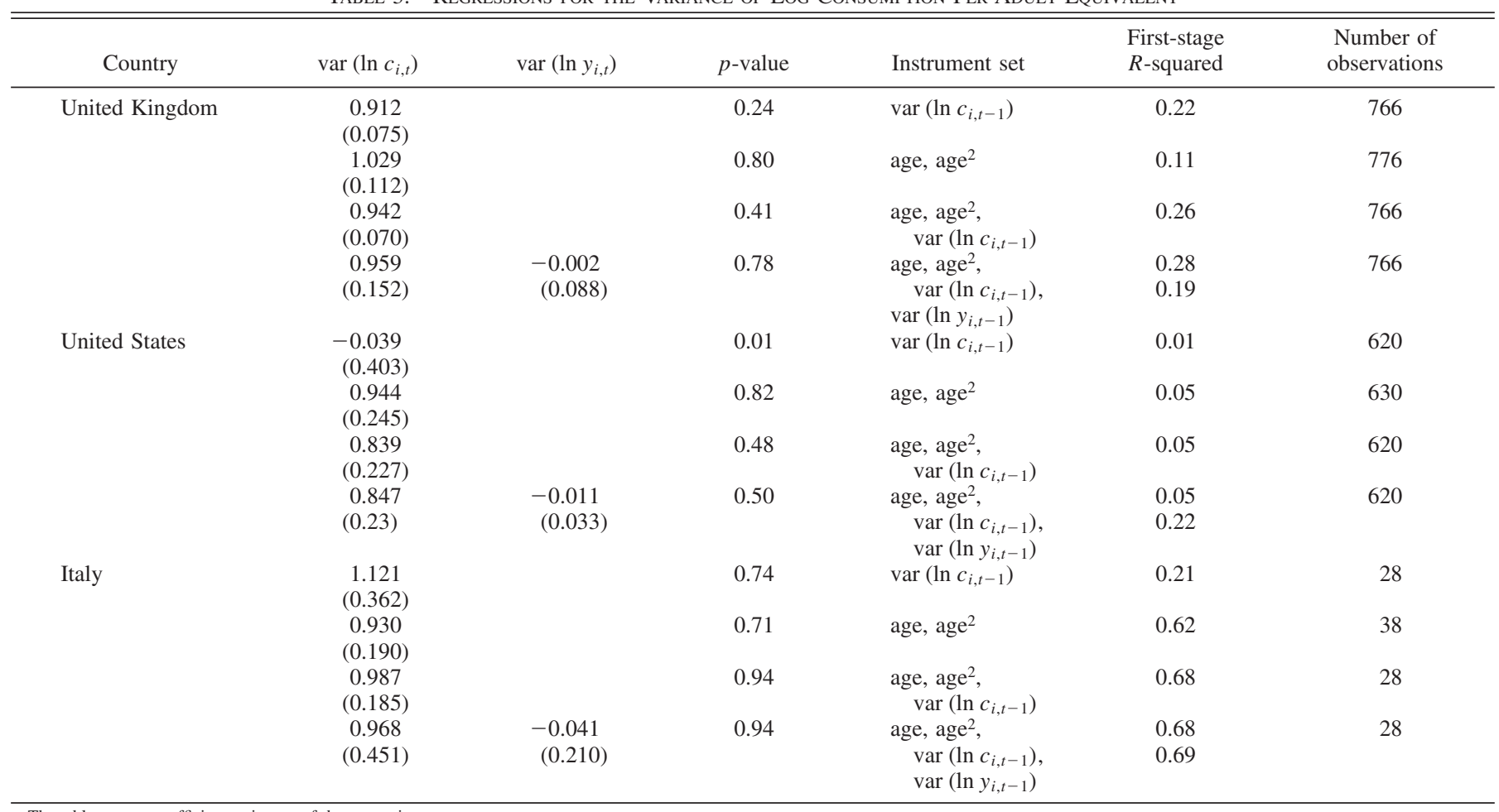

The table reports coefficient estimates of the regression

$$
\operatorname{var}\left\lfloor\ln \left(c_{i, t+1} / P_{i, t+1}\right)+\sigma \ln \left(P_{i, t+1} / N_{i, t+1}\right)\right\rfloor=\varpi_{0}+\pi \operatorname{var}\left\lfloor\ln \left(c_{i, t} / P_{i, t}\right)+\sigma \ln \left(P_{i, t} / N_{i, t}\right)\right\rfloor+\varpi_{t+1} .
$$

$P$ denotes the number of adult equivalent, $N$ is family size, and $\sigma$ is the intertemporal elasticity of substitution. We assign a weight of 1 to the first adult, 0.8 to any additional adult, and 0.25 to each child, $P=$ $1+0.8$ (Adults -1 ) +0.25 Children, and set $\sigma=0.8$. The $p$-value refers to the test that $\pi$ is significantly different from 1 . All regressions are estimated by instrumental variables. The two $R$-squared in the fourth specification for each country refer to the first-stage regressions for the lagged variance of consumption and for the lagged variance of income, respectively. Standard errors are reported in parenthesis.

much closer to 1 . In no case can the hypothesis that $\pi=1$ be rejected at conventional significance levels. The only exception is the first regression for the United States. Note, however, that in this case the correlation between $\operatorname{var}\left(\ln c_{i t}\right)$ and $\operatorname{var}\left(\ln c_{i t-1}\right)$ is very low, resulting in a value for $R$-squared in the first-stage regression of only 0.01 . The results reported in table 3 are again robust to the exclusion from the sample of the oldest cohorts and to the inclusion of the lagged income variance as an additional regressor.

In table 4 , we compute marginal utility using the specification

$$
U(c, z)=\frac{c_{i, t}^{1-\sigma^{-1}}}{1-\sigma^{-1}} \exp \left(-\theta^{\prime} z_{i, t}\right)
$$

in which $z$ includes family size and indicator variables for blue-collar workers, heads out of employment, full-time working spouse, and more than two income recipients. This specification of the instantaneous utility function has been estimated by Attanasio and Weber $(1993,1995)$ on the same FES and CEX cohort data. Thus, we can readily use their estimates of $\theta$ to compute marginal utility. ${ }^{21}$ The actual values of the estimates and the details about the construction

${ }^{21}$ If the intertemporal elasticity of substitution, $\sigma$, is constant across households, it represents only a factor of proportionality that does not affect the regression results. Attanasio and Weber (1993) also consider seasonal shifts of preferences. Because these shifts are assumed to be of marginal utility are described in appendix A. We do not attempt to fit a flexible Euler equation to Italian data. Due to the few surveys and the fact that we work with annual data, the number of observations is much lower than it is in the other two countries. The point estimate of $\pi$ for the United Kingdom and the United States in Table 4 are again very close to unity, regardless of the instrument set. Given also the small standard errors of the estimate, in no case can we reject the hypothesis that $\pi=1$. The point estimates obtained by dropping the retired do not show again appreciable differences as well as those including the lagged income variance.

Our results show that an important implication of the lifecycle model-namely that the coefficient on the lagged variance in equation (9) is equal to unity-is not rejected by the data. It may seem surprising that this result holds for all different measures of marginal utility. However, one should consider that our instrumental-variable strategy and the fact that we are working with isoelastic specifications would give us consistent estimates of the parameter of interest as long as the instruments used are uncorrelated with the omitted variables (for instance, with the cross-sectional variance of adult equivalents).

multiplicative and constant across consumers, they do not affect the computation of the cross-sectional variance. 
Table 4.-Regressions for the Variance Computed with Flexible Preference Specification

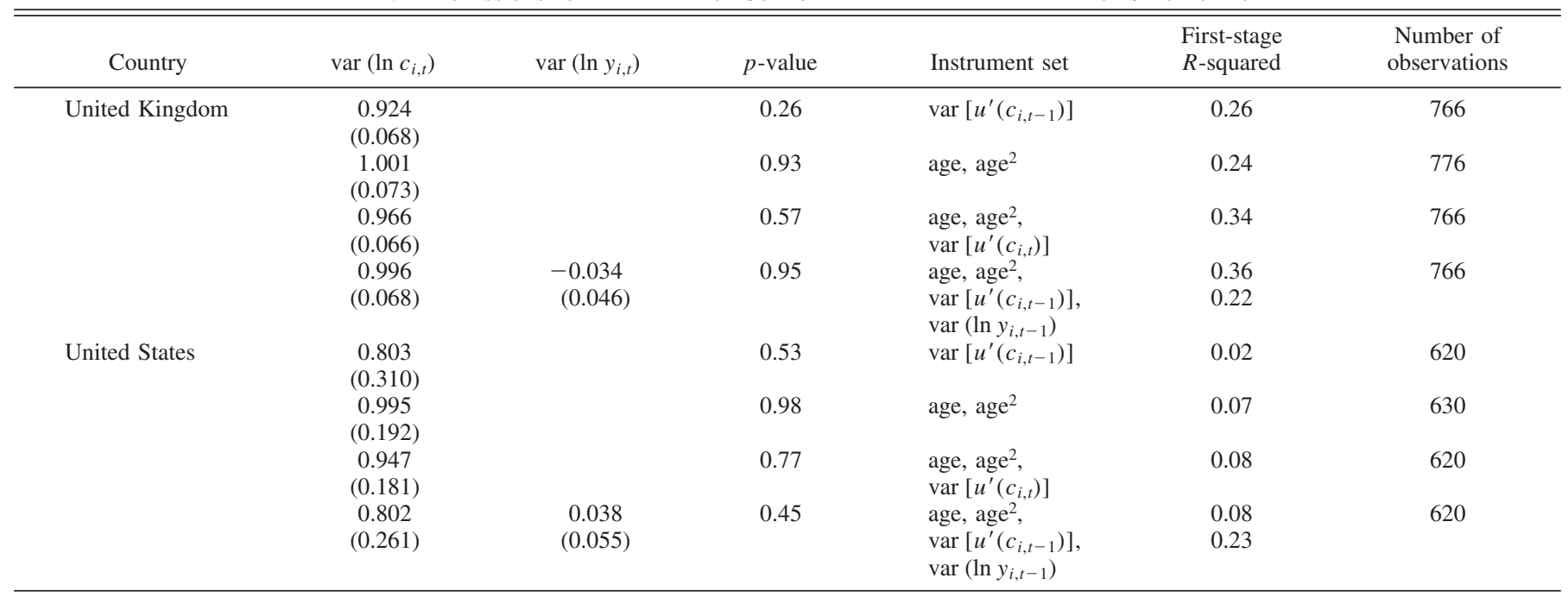

The table reports regressions of var $\left[\ln \left(c_{i, t+1}\right)+\theta^{\prime} z_{i, t+1}\right]=\varpi_{0}+\pi \operatorname{var}\left[\ln \left(c_{i, t}\right)+\theta^{\prime} z_{i, t}\right]+\varpi_{t+1}$. The $z$ variables include demographic characteristics and labor market indicators. As described in appendix A, we draw from existing studies to impute values for the $\theta$ parameters. The $p$-value refers to the test that $\pi$ is significantly different from 1 . All regressions are estimated by instrumental variables. The two $R$-squared in the fourth specification for each country refer to the first-stage regressions for the lagged variance of consumption and for the lagged variance of income, respectively. Standard errors are reported in parenthesis.

\section{Conclusions}

The lifecycle hypothesis predicts that the cross-sectional variance of the marginal utility of consumption is equal to its own lag plus a constant and a random component. Using fairly general preference specifications and auxiliary assumptions about the nature of the random component, we provide an explicit test of this hypothesis. Our approach circumvents the necessity to identify a pure age profile of the cross-sectional variance of consumption and yields a well-specified statistical test. On the other hand, the test we propose is valid only under specific assumptions about the shocks of the Euler equation for consumption.

Cohort data for the United Kingdom, the United States, and Italy provide strong support for the hypothesis that the coefficient of the lagged cross-sectional variance of marginal utility is equal to 1 . That is, the joint hypothesis that the theoretical restrictions on the evolution of the crosssectional variance and the auxiliary identification assumptions we use are consistent with the data.

As we have repeatedly stressed, our test does not assume certainty equivalence. Rather, we are able to confront with the data a flexible representation of preferences that allows for departures from certainty equivalence, dependence on family size, labor supply, and other demographic variables. This increased generality, however, has a price: we loose the ability to relate the evolution of the cross-sectional variance of consumption to the evolution of the cross-sectional variance of earnings. This relation has proven to be particularly useful in welfare comparisons among households.

Blundell and Preston (1998) study under which conditions consumption is a better indicator of welfare inequality than income. They note that, in the lifecycle model, crosssectional comparisons of consumption inequality are informative about welfare inequality only on restrictive assumptions that concern the form of the utility function. They also show that under certainty equivalence the evolution of the variance of consumption should reflect permanent-income innovations, whereas the variance of earnings should reflect permanent as well as transitory changes in uncertainty. Our study is complementary to theirs in that it shows that one of the most general implications of individuals' intertemporal choices for the evolution of the cross-sectional variance is borne out by the data.

Dropping certainty equivalence, only simulation analysis can give an idea of the structural relation between the cross-sectional variances of earnings and consumption (examples of such simulation models are in Hubbard, Skinner, and Zeldes (1995)). In principle, these models could simulate the consumption behavior of a generation and compute the evolution of the cross-sectional variance of marginal utility. By changing the stochastic properties of the incomegenerating process, it would then be possible to study the relation between income and consumption inequality. We regard this as an interesting topic for future research.

\section{REFERENCES}

Attanasio, Orazio P., and Guglielmo Weber, "Consumption Growth, the Interest Rate and Aggregation," Review of Economic Studies 60 (July 1993), 631-649.

"Is Consumption Growth Consistent With Intertemporal Optimization? Evidence from the Consumer Expenditure Survey," Journal of Political Economy 103 (December 1995), 1121-1157.

Banks, James, and Paul Johnson, How Reliable Is The Family Expenditure Survey Data. Trends in Incomes and Expenditure over Time. (London: Institute for Fiscal Studies, 1997).

Blundell, Richard, and Ian Preston, "Consumption Inequality and Income Uncertainty," Ouarterly Journal of Economics 113 (May 1998), 603-640.

Deaton, Angus, and Christina Paxson, "Intertemporal Choice and Inequality," Journal of Political Economv 102 (June 1994), 437-467.

Hubbard, Glenn R., Jonathan Skinner, and Stephen P. Zeldes, "Precautionary Saving and Social Insurance," Journal of Political Economv 103 (April 1995), 360-399. 


\section{APPENDIX A}

\section{Seasonal Adjustment}

In the FES and in the CEX, consumption is observed at a frequency higher than annual. In the FES, consumption refers to the two-week period over which the household is interviewed; in the CEX, it measures the flow of expenditure in each of the three months preceding the interview. This feature of the data makes seasonal adjustment indispensable: we must avoid mixing seasonal variations with the cross-sectional variability of consumption.

Because in the FES each household is interviewed only once, seasonal adjustment is easier. This is performed using a simple log-linear model of monthly seasonality. In the CEX, each household provides up to twelve observations. There are two pitfalls. On the one hand, we do not want to mix seasonal with genuine cross-sectional variability; on the other, we want to avoid having the same household providing more than one observation within each period cell. ${ }^{22}$ If the interviews were synchronized, it would be easy to construct annual (or quarterly) consumption figures and use those in computing means and variances by year. Given the overlapping nature of the sample, however, we rely on an alternative procedure. Among several alternatives, we report results for the simplest: for each household, we consider only the first monthly observation. (We use the first rather than the last observation to minimize the influence of nonrandom attrition.) The data are then adjusted for seasonality using the same model as in the case of the FES data.

\section{Parameters of the Euler Equations Used to Compute the Marginal Utility}

The parameters used in the constructions of the marginal utility of consumption are taken from Attanasio and Weber (1993, 1995). If

$$
U(c, z)=\frac{c_{i, t}^{1-\sigma^{-1}}}{1-\sigma^{-1}} \exp \left(\theta^{\prime} z_{i, t}\right)
$$

\footnotetext{
${ }^{22}$ This is because, if a household provides observations in different cells,
} problems arise in the appropriate choice of instruments. the Euler equation for consumption is

$$
\Delta \ln \left(c_{i, t+1}\right)=\text { constant }+\sigma\left(1+r_{t+1}^{k}\right)+\theta^{\prime} \Delta z_{i, t+1}+\epsilon_{i, t+1} .
$$

Further assuming that $z$ includes demographic as well as labor supply variables and using quarterly cohort data from the FES (1970-1987), Attanasio and Weber (1993) estimate

$$
\begin{aligned}
\Delta \ln \left(c_{i, t+1}\right)= & 0.747 r_{t+1}+0.478 \Delta w c h+0.2201 \Delta \text { adults } \\
& +0.7086 \Delta m a+0.0063 \Delta \text { children } \\
& +0.0446 \Delta \text { young children }-0.9415 \Delta \text { oow } \\
& +0.2725 \Delta w w+\text { seasonal dummies, }
\end{aligned}
$$

where oow is a dummy for the household head out of work, $m a$ is a dummy indicating more than two adults, $w c h$ is a dummy for white-collar workers, $w w$ is a dummy for working spouse, and young children are those of preschool age $(0-5)$.

For details and standard errors, see Attanasio and Weber (1993, column 2 of table 1 of their appendix).

For the United States, Attanasio and Weber (1995) use quarterly cohort data derived from the CEX from 1981:3 to 1990:4 and estimate

$$
\begin{aligned}
\Delta \ln \left(c_{i, t+1}\right)= & 0.341 r_{t+1}+1.172 \Delta \text { family size } \\
& -0.539 \Delta \text { children }-1.551 \Delta w w \\
& +-2.578 \Delta \ln w l-2.239 \Delta \text { single } \\
& + \text { seasonal dummies }
\end{aligned}
$$

where children is the household members between the ages of 0 and 15 , $w w$ is a dummy for the wife working full-time, and $w l$ is the wife's annual hours of leisure. (See Attanasio and Weber 1995, table 3, column 3.)

We ignore the real interest-rate term, which is only a factor of proportionality and does not affect the regression results. We also ignore the seasonal dummies because they do not affect the computation of the cross-sectional variance. These parameters allow the straightforward computation of $\left[\ln \left(c_{i, t}\right)+\sigma \theta^{\prime} z_{i, t}\right]$. 
This article has been cited by:

1. Risto Herrala 10, 47. [CrossRef]

2. Tullio Jappelli , Luigi Pistaferri . 2006. Intertemporal Choice and Consumption MobilityIntertemporal Choice and Consumption Mobility. Journal of the European Economic Association 4:1, 75-115. [Abstract] [PDF] [PDF Plus] 